\title{
Comparação das pressões respiratórias máximas em idosos que praticam exercícios no solo e água
}

\author{
Comparison of maximal respiratory pressures in elderly \\ subjects who practice exercises in soil and water \\ Comparación de la fuerza muscular respiratoria en adultos \\ mayores que hacen ejercicio en el suelo y agua
}

Simone Mader Dall Agnol

Thaisa Iohn da Silva

Denise Barth Rebesco

André de Camargo Smolarek

William Cordeiro de Souza

Meiriélly Furmann

Luis Paulo Gomes Mascarenhas

RESUMO: O objetivo do estudo foi comparar a força muscular respiratória em idosos saudáveis praticantes de exercícios em dois meios, solo e água. A amostra foi composta por 20 idosos de ambos os gêneros, que foram divididos em dois grupos, GS (Grupo-Solo) e GA (Grupo-Água). As avaliações das pressões inspiratórias máximas (PImáx) e pressões expiratórias máximas (PEmáx) foram realizadas através do aparelho manovacuômetro manual. Os resultados demonstraram não haver alterações significativas na musculatura respiratória de ambos os grupos.

Palavras-chave: Fisioterapia; Envelhecimento; Exercícios Respiratórios. 
ABSTRACT: The objective of this study was to compare respiratory muscle strength in healthy elderly exercise practitioners in two media, soil and water. The sample consisted of 20 elderly of both genders, who were divided into two groups, GS (Soil Group) and GA (Water Group). The maximal inspiratory pressures (MIP) and maximal expiratory pressures (MEP) were evaluated using the manual manometer instrument. The results showed no significant changes in the respiratory muscles of both groups.

Keywords: Physiotherapy; Aging; Respiratory Exercises.

RESUMEN: El objetivo del estudio fue comparar la fuerza muscular respiratoria en ancianos sanos practicantes los ejercicios en dos maneras, el suelo y el agua. La muestra fue constituida por 20 ancianos de ambos sexos, que fueron divididos en dos grupos, GS (Grupo Suelo) y GA (Grupo Agua). Las evaluaciones de la presión inspiratoria máxima (PIM) y la presión espiratoria máxima (PEM) se realizaron usando aparato manómetro de vacío manual. Los resultados no mostraron cambios significativos en los músculos respiratorios de ambos grupos.

Palabras clave: Fisioterapia; Envejecimiento; Ejercicios de Respiración.

\section{Introdução}

O envelhecimento é um processo natural de cada indivíduo, sendo que este se manifesta diferente de pessoa para pessoa, uma vez que cada um envelhece em ritmos variados, ainda que com a mesma idade cronológica (Barbon, Wiethölter, \& Flores, 2016). De acordo com Pereira, Batista, Fuly, Junior, \& Silva (2014), o declínio do sistema cardiorrespiratório inicia-se aos vinte anos e acelera por volta dos setenta anos, com a redução da elasticidade pulmonar e déficits de funcionalidade.

Com o envelhecimento, algumas alterações estruturais no aparelho respiratório são evidentes; estas compreendem uma caixa torácica enrijecida, diminuição na elasticidade pulmonar bem como da força dos músculos respiratórios. A capacidade vital decresce enquanto que o volume residual aumenta. Este processo também acarreta diminuição da ventilação pulmonar, da elasticidade dos alvéolos e subtração da capacidade vital (Fechine, \& Trompieri, 2015).

As alterações na função pulmonar relacionadas com a idade são clinicamente relevantes, visto que uma função pulmonar precária é associada a taxas elevadas de mortalidade. 
Uma pessoa idosa utiliza $70 \%$ da elasticidade total de sua parede torácica, enquanto um jovem utiliza apenas $40 \%$ do seu máximo (Ide, Caromano, Dip, \& Guerino, 2007).

Em geral, o processo de envelhecimento está associado a um grande número de modificações fisiológicas que reduzem o processo natural de defesa de reserva das vias aéreas (Tran, Rajwani, \& Berlin, 2018).

Sendo assim, a atividade física é a chave para promover uma boa saúde e uma melhor qualidade de vida do idoso. A prática regular traz muitos benefícios para a saúde e previne patologias respiratórias; tanto os exercícios realizados no ambiente terrestre, quanto no aquático, apresentam inúmeras vantagens podendo estar associadas à melhora na capacidade aeróbica, nas trocas gasosas, reeducação respiratória, auxílio no retorno venoso, aumentando, com isso, a capacidade do sistema pulmonar (Costa, \& Jarnani, 2001).

A fisioterapia aquática utiliza exercícios com o intuito de prevenir, manter, retardar e melhorar disfunções físicas e psicológicas do envelhecimento (Ramos, \& Ferreira, 2014). Esta terapia oferece inúmeros benefícios para os idosos, como a diminuição da dor, aumento ou manutenção das amplitudes articulares, força muscular, condição cardiovascular, capacidade vital e equilíbrio; controle do peso corporal, promoção do relaxamento, manutenção de atividades funcionais além da socialização e recreação (Assis, Junior, Santos, \& Navarro, 2011).

Kruel, Cortjens, Pinto, \& Alberton (2006) destacam que, ao entrar na água, o indivíduo sofre alterações fisiológicas que podem ser ocasionadas pela temperatura da água, pela ação da pressão hidrostática e pela redução do peso hidrostático.

Carregaro, e Toledo (2008) descrevem que os efeitos fisiológicos proporcionados pela água são amplos e envolvem respostas cardíacas, respiratórias, renais e músculo-esqueléticas. Assim como acontece em boa parte do organismo, o fluxo sanguíneo no pulmão também aumenta devido à elevação da pressão sanguínea; esta reação contribui para uma maior troca gasosa, devido ao aumento de sangue na circulação pulmonar.

Em imersão, ocorre um trabalho respiratório em decorrência da pressão hidrostática, a qual exerce carga para a contração do diafragma; além disso, essa mesma carga auxilia na elevação do diafragma, colaborando no processo da expiração (De Almeida Fagundes, \& Da Silva, 2017).

Com base nesse contexto, o presente estudo teve por objetivo comparar a força muscular respiratória em idosos saudáveis praticantes de exercícios em dois meios, solo e água. 


\section{Métodos}

A amostra intencional do presente estudo foi composta por 20 idosos de ambos os gêneros, com idade entre 60 e 78 anos. Foram 10 participantes que realizavam atividade: no solo, GS (Grupo-solo); e 10 participantes que realizavam atividade na água, GA (Grupo-água). O tamanho da amostra foi calculado com base em um erro probabilístico de 0,05 e power de 0,95 apresenta um tamanho do efeito $\mathrm{dz}=0,8$. Todos os participantes foram previamente informados em relação aos procedimentos aos quais seriam submetidos. Em seguida, assinaram um termo de consentimento livre e esclarecido (TCLE). O projeto foi aprovado pelo Comitê de Ética em Pesquisa da Universidade Estadual do Centro-Oeste (UNICENTRO), conforme parecer n. ${ }^{\circ}$ 999.011. Foram excluídos do estudo indivíduos com idade inferior a informada, portadores de patologias pulmonares crônicas diagnosticadas, idosos não praticantes das atividades informadas, tabagistas e hipertensos não controlados, portadores de patologias neurológicas ou neuromusculares, e indivíduos que se recusaram a assinar o termo de consentimento (TCLE).

Os indivíduos foram divididos por conveniência em dois grupos. O grupo-solo (GS) realizava há mais de 6 meses exercícios de musculação envolvendo grupos musculares de membros superiores, inferiores e de tronco. Ao final de cada treino os participantes realizavam alongamentos ativos de toda a musculatura recrutada. O grupo-água (GA) praticava há mais de 6 meses exercícios de fortalecimento de membros superiores, inferiores e tronco com auxílio de flutuadores, bem como exercícios aeróbicos como marcha estática associada ao movimento ativo de membros superiores. Estes foram executados em uma piscina cuja média da altura é de $1,25 \mathrm{~cm}$. Segundo Canderolo, e Caromano, (2007) e Elias, Gonçalves, Moraes, Formaggio, e Fernandes, (2012), a altura média indicada para realizações de atividades na água é de $1,10 \mathrm{~cm}$ a $1,30 \mathrm{~cm}$ de profundidade.

Ambos os grupos realizavam suas respectivas atividades por pelo menos seis meses, três vezes na semana, com duração média de 60 minutos. Os exercícios foram realizados com intensidade progressiva, de acordo com a habilidade de cada indivíduo (Ide, Caromano, Dip, \& Guerino, 2007). A pesquisa foi realizada nas dependências da Clínicas Integradas Guairacá, de propriedade da Faculdade Guairacá, Guarapuava, PR.

Para caracterização da amostra, coletaram-se os dados de massa corporal e estatura. Na mensuração da massa corporal, o avaliado deveria se posicionar em pé, de costas para escala da balança, usando o mínimo de roupa possível (Petroski, 2011). Foi utilizada uma balança digital da marca Techline, devidamente calibrada, com graduação de 100 gramas e escalas variando de 0 a $180 \mathrm{Kg}$. 
A mensuração da estatura foi identificada pelo maior valor entre o vértex e a região plantar obedecendo ao plano de Frankfurt (Petroski, 2011). A estatura foi verificada através de uma trena flexível marca Sanny Medical Sparrett, resolução de 0,1 mm, fixada na parede lisa, com 3 metros e graduação de $0,1 \mathrm{~cm}$ com o zero coincidindo com o solo. Após a obtenção desses dados foi calculado o Índice de Massa Corporal (IMC) utilizando-se a seguinte formula: IMC= Peso Corporal/Estatura².

A avaliação da força da musculatura respiratória foi realizada por meio do aparelho manovacuômetro analógico da marca comercial Médica, em que cada participante realizou as medidas das pressões inspiratória máxima (PImáx) e pressão expiratória máxima (PEmáx) (Sarmento, 2010). O participante foi atendido de forma individual, sendo que, entre a troca de participantes, o bocal foi removido e descartado. A intervenção inteira foi assistida pelo mesmo pesquisador.

O participante foi convidado a permanecer sentado, e com os pés apoiados no chão, utilizando um clipe nasal. Este foi orientado a segurar firmemente no conector bocal, pressionandoo contra os lábios. Na realização da pressão inspiratória máxima (PImáx), foi solicitado ao idoso exalar todo o volume pulmonar até o volume residual (VR) e, após, realizar um esforço inspiratório máximo, sustentando a pressão por aproximadamente um segundo. Na realização da medição da pressão expiratória máxima (PEmáx), foi solicitado ao idoso insuflar os pulmões até a capacidade pulmonar total (CPT) e, após, realizar uma expiração forçada, sustentando a pressão máxima por aproximadamente um segundo. Os procedimentos foram repetidos em três tentativas, com intervalo de um minuto entre cada uma, tanto para a PImáx quanto para a PEmáx, sendo considerado para o estudo o maior valor obtido nas três tentativas de cada pressão colhida. Caso o último dos três valores mensurados fosse o maior, foi realizada uma nova repetição, até que a última medida não tenha sido a maior obtida. A postura adotada foi mantida durante toda a execução do teste (Bellinetti, \& Thomson, 2006).

A graduação das pressões respiratórias é de $-120 \mathrm{cmH} 2 \mathrm{O}$ a $+120 \mathrm{cmH} 2 \mathrm{O}$, com escala de 4 em 4 cmH2O, sendo os valores negativos correspondentes às pressões inspiratórias, enquanto os valores positivos correspondem às pressões expiratórias. Estes valores são descritos pela equação de Black, \& Hyatt (1969), cujos valores de Pimáx partem do VR (volume residual), e da PEmáx parte da CPT (Capacidade Pulmonar Total) (Presto, B. \& Presto, L.D.N., 2009). O registro da força dos músculos respiratórios na PImáx e PEmáx foi em cmH2O, os dispositivos eletrônicos medem com maior precisão e confiabilidade do que os não eletrônicos (Deturk, \& Cahalin, 2007). 
Entretanto, é possível obter também medições precisas e confiáveis, usando-se esses objetos menos custosos se for observada com cuidado a magnitude do ponteiro durante as medições inspiratórias e expiratórias da força muscular ventilatória (Deturk, \& Cahalin, 2007). Os valores espirométricos foram expressos como a porcentagem do valor previsto normal para a população brasileira (Pereira, Sato, \& Rodrigues, 2007).

Para verificar a normalidade dos dados, foi realizado o teste de Shapiro-Wilk. Após, calculou-se a média e desvio-padrão dos dados. Na comparação entre os grupos GS e GA, aplicouse o teste t para amostras pareadas. Foi estipulado o nível de significância de 0,05 . O tamanho do efeito foi calculado pelo teste de Cohen na comparação entre grupo solo e grupo agua e classificado de acordo: baixo efeito $d>0,2$; efeito moderado $0,2<d>0,5$; grande efeito $d<0,5$. Os dados foram analisados de forma descritiva e inferencial através do programa estatístico SPSS 16.0 for Windows.

\section{Resultados}

A tabela 1 apresenta os dados de idade e medidas antropométricas (massa corporal, estatura e IMC) para a caracterização da amostra, e é possível observar que não foram encontradas diferenças significativas nas comparações entre grupos nas variáveis analisadas.

Tabela 1. Caracterização da amostra $(n=20)$

\begin{tabular}{ccccc}
\hline Variáveis & GS $(\mathbf{n = 1 0})$ & GA $(\mathbf{n}=\mathbf{1 0})$ & T & p \\
Idade (anos) & $70,9 \pm 6,5$ & $66,9 \pm 5,9$ & 1,424 & 0,188 \\
Massa Corporal $(\mathrm{kg})$ & $77,55 \pm 10,46$ & $74,55 \pm 10,37$ & 0,667 & 0,260 \\
Estatura $(\mathrm{m})$ & $1,63 \pm 11,5$ & $1,61 \pm 6,3$ & 0,502 & 0,313 \\
IMC $\left(\mathrm{kg} / \mathrm{m}^{2}\right)$ & $29,19 \pm 4,60$ & $28,43 \pm 3,38$ & 0,422 & 0,341 \\
\hline
\end{tabular}

Nos gráficos 1 e 2, a seguir, são representados os valores médios de PImáx e PEmáx dos grupos GS (PImáx -81,5 $\mathrm{cmH}_{2} \mathrm{O} \pm-37,71$ e PEmáx $+86,5 \mathrm{cmH}_{2} \mathrm{O} \pm+22,61$ ) e GA (PImáx $68,6 \mathrm{cmH}_{2} \mathrm{O} \pm-28,09$ e PEmáx $+91,5 \mathrm{cmH}_{2} \mathrm{O} \pm+22,11$ ); e pode-se perceber que não houve diferenças significativas entre ambos. Contudo observou-se um tamanho do efeito moderado PImáx $(\mathrm{d}=-0,39 \mathrm{ES}=-0,19)$ e para PEmáx $(\mathrm{d}=-0,22 ; \mathrm{ES}=-0,11)$ em favor do meio aquático. A PImáx indica a força do músculo diafragma, enquanto a PEmáx é uma medida que indica a força dos músculos abdominais e intercostais (Costa, Sampaio, Lorenzzo, Jamami, \& Damaso, 2003). 


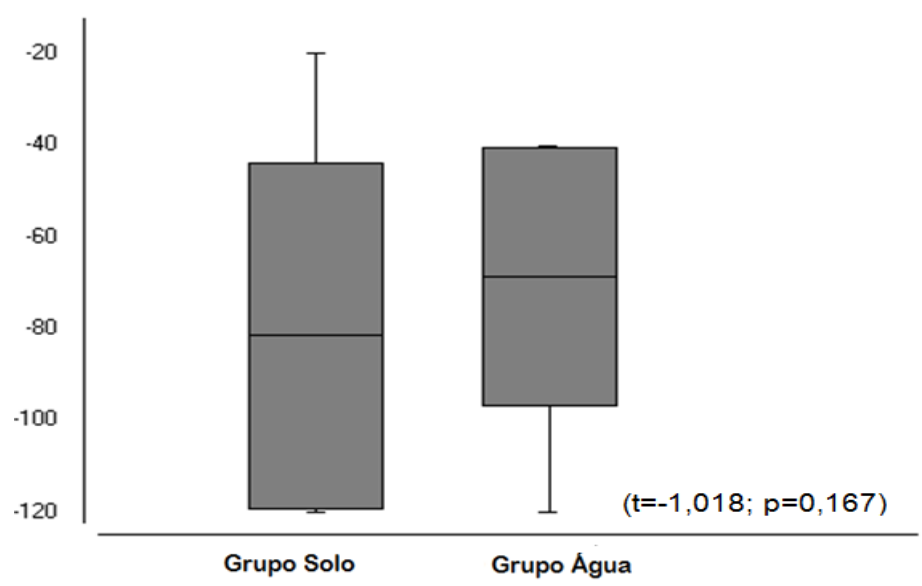

Gráfico 1. Valores de PImáx $\left(\mathrm{cmH}_{2} \mathrm{O}\right)$ dos grupos solo e água

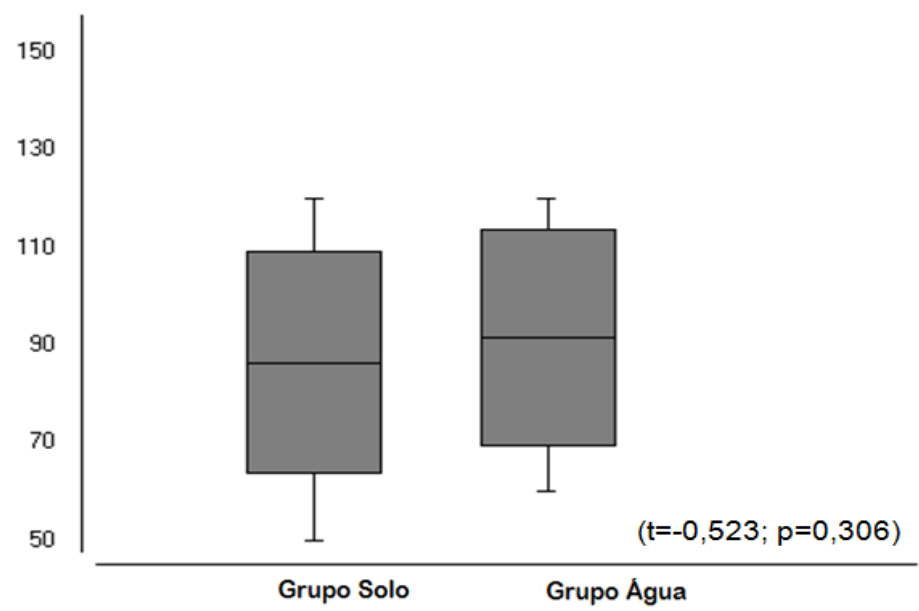

Gráfico 2. Valores de PEmáx $\left(\mathrm{cmH}_{2} \mathrm{O}\right)$ dos grupos solo e água

\section{Discussão}

O presente estudo buscou como objetivo comparar a força muscular respiratória em idosos saudáveis praticantes de exercícios em dois meios, solo e água. Através dos resultados encontrados não foram observadas diferenças significativas nas variáveis de PImáx e PEmáx, em ambos os grupos avaliados.

Os valores previstos para homens entre 60 a 69 anos na PImáx são de $-104,34 \mathrm{cmH}_{2} \mathrm{O}$ e para a PEmáx de $+113,70 \mathrm{cmH}_{2} \mathrm{O}$. Na faixa etária de 70 a 80 anos, os valores de PImáx e PEmáx são respectivamente $-93,7 \mathrm{cmH}_{2} \mathrm{O}$ e $+102,3 \mathrm{cmH}_{2} \mathrm{O}$. Contudo, nas mulheres, a PImáx entre 60 a 69 anos é de $-78,7 \mathrm{cmH}_{2} \mathrm{O}$ e $+76,1 \mathrm{cmH}_{2} \mathrm{O}$ para a PEmáx e na faixa etária de 70 a 90 anos os valores previstos para PImáx e PEmáx são respectivamente de $-73,3 \mathrm{cmH}_{2} \mathrm{O}$ e $+69,4 \mathrm{cmH}_{2} \mathrm{O}(\mathrm{Costa}$, Gonçalves, Lima, Ike, Cancelliero, \& Montebelo, 2010). 
Comparando esses valores aos do presente estudo, observa-se que os valores de PImáx e PEmáx foram menores; sendo assim, os valores encontrados no presente estudo foram considerados satisfatórios.

De acordo com Cruz, Fernandes, Reis, Policarpo, e Filho (2010), durante o processo de envelhecimento, ocorre um aumento da adiposidade, principalmente na região visceral, e estaria correlacionado com a redução das pressões respiratórias, relacionando com a restrição de movimento de expansibilidade torácica e também com a redução de força muscular. No presente estudo, observou-se que as médias do IMC tanto no GS, quanto GA resultaram em sobrepeso; provavelmente esse fator deve ter afetado nos valores das pressões respiratórias.

Fato interessante encontrado na literatura é que a força muscular respiratória diminui aproximadamente $8-10 \%$ por década a partir dos 40 anos, segundo pesquisa realizada com 120 indivíduos saudáveis (60 homens e 60 mulheres), com idades entre 20 e 80 anos, residentes em São Carlos, SP(Costa, Gonçalves, Lima, Ike, Cancelliero, \& Montebelo, 2010).

Gonçalves, Tomaz, Cassiminho, e Dutra (2006), em seu estudo realizado na Cidade de Santa Maria, RS, avaliaram e compararam valores de PImáx e PEmáx em idosas a partir de 65 anos, praticantes regulares de atividades físicas duas vezes por semana e sedentárias. Os pesquisadores verificaram que os sujeitos praticantes de atividade física apresentaram um aumento significativo nas pressões respiratórias máximas, quando comparadas aos que não praticavam, porém ambos os grupos obtiveram valores inferiores ao predito. Discorda-se do presente estudo, em que se observou valores acima do previsto nos indivíduos estudados.

Nascimento, Trindade, Oliveira, Sousa, \& Abrahin (2013) também compararam as pressões respiratórias de idosos, porém estes foram classificados em grupos praticantes de exercícios resistidos e grupo sedentário. Assim como visto do atual estudo, estes autores verificaram que o grupo de idosos praticantes de exercícios apresentaram valores superiores ao esperado para idade, diferente do encontrado no grupo de idosos sedentários, os quais apresentaram valores inferiores ao esperado para a idade.

Freitas, Araújo, e Alves, (2012), realizando estudo com indivíduos tabagistas e não tabagistas de ambos os sexos com idade acima de 60 anos, não encontraram diferenças significativas nos valores de PImáx e PEmáx. Assim também o presente estudo não encontrou diferenças significativas entre os grupos estudados, cabendo mencionar que, na presente pesquisa, não foram incluídos os idosos fumantes. 
Segundo Parreira, et al. (2007), que realizaram estudo com 103 indivíduos de Belo Horizonte, Itabira e Sete Lagoas, MG, com idade entre 20 e 80 anos, verificaram que a PImáx e PEmáx são dependentes, não apenas da força dos músculos respiratórios, mas também do volume pulmonar, em que são realizadas as medidas, e do correspondente valor da pressão de retração elástica do sistema respiratório.

Os indivíduos que realizaram exercícios no meio aquático apresentaram valores superiores em relação aos praticantes de exercício no solo. Esses melhores resultados obtidos pelo GA provavelmente são causados pelos efeitos das propriedades térmicas e mecânicas da água sobre o indivíduo imerso, que maximizaram o exercício, além dos efeitos psicológicos proporcionados pela terapia aquática. Sabe-se também que o efeito térmico da água é responsável por uma melhora na complacência dos tecidos moles e articulações. O calor também promove relaxamento, o que auxilia na melhora das amplitudes de movimento articular. Todos estes efeitos são maximizados quando o calor é aplicado de forma constante, e em todo o organismo, como o proporcionado pela terapia em piscina terapêutica (Teixeira, Pereira, \& Rossi, 2007). Estes efeitos, exercidos sobre as articulações da caixa torácica e cintura escapular, podem ter sido responsáveis pela melhora encontrada no GA.

Independentemente do mecanismo, sugere-se que o aumento da força muscular respiratória pode contribuir para melhorar a capacidade de exercício e diminuir o risco de infecções respiratórias e hospitalizações devido ao ganho de força expiratório que gera maior eficácia de tosse (Souza, et al., 2014).

Diante dos resultados apresentados é importante considerar que indivíduos ativos apresentaram médias maiores de pressões respiratórias que a média esperada para a idade, reforçando-se, assim, que a prática regular de atividade física reduz a perda de força muscular respiratória advinda do processo de envelhecimento, sendo o meio aquoso uma opção salutar.

Nesse sentido, o presente estudo se destaca, pois se compreende haver possibilidades de suprimir alguns efeitos advindos do envelhecimento por meio de ações simples e de baixo custo, fomentando assim a participação dessa população em atividades comunitárias enquanto estratégia de promoção da saúde.

O presente estudo apresentou algumas limitações como escassez de estudos similares na comparação dos grupos analisados, além do mais se enfrentou dificuldade em encontrar um número expressivo de participantes. 


\section{Conclusão}

Os dados apresentados não demonstraram resultados estatisticamente significativos entre os grupos analisados; contudo, os valores das pressões respiratórias medidos na amostra superaram os valores previstos pela idade, tanto para o grupo-solo como para o grupo-água.

\section{Referências}

Assis, R. S., Junior, L. F. S. D. S., Santos, L. R., \& Navarro, A. C. (2011). A hidroginástica melhora o condicionamento físico dos idosos. Revista Brasileira de Prescrição e Fisiologia do Exercício, 1(5), 62-75. Recuperado em 17 março, 2017, de: http://www.rbpfex.com.br/index.php/rbpfex/article/view/49/48.

Barbon, F. J., Wiethölter, P., \& Flores, R. A. (2016). Alterações celulares no envelhecimento humano. Journal of Oral Investigations, 5(1), 61-65. Recuperado em 17 março, 2018, de: https://seer.imed.edu.br/index.php/JOI/article/view/1379/pdf.

Bellinetti, L. M., \& Thomson, J. C. (2006). Avaliação muscular respiratória nas toracotomias e laparotomias superiores eletivas. Jornal Brasileiro de Pneumologia, 32(2), 99-105. Recuperado em 03 junho, 2016, de: http://www.scielo.br/scielo.php?script=sci_arttext\&pid=S180637132006000200004.

Black, L, F., \& Hyatt, R. E. (1969). Maximal respiratory pressures: normal values and relationship to age and sex. American Review of Respiratory Disease, 99(5), 696-702. Recuperado em 11 setembro, 2016, de: https://www.ncbi.nlm.nih.gov/pubmed/5772056.

Canderolo, M. J., \& Caromano, F. A. (2007). Elaboração, aplicação e avaliação de um programa de ensino de adaptação ao meio aquático para idosos. Acta Fisiátrica, 14(3), 170-175. Recuperado em 11 junho, 2016, de: http://www.actafisiatrica.org.br/detalhe_artigo.asp?id=206.

Carregaro, R. L., \& Toledo, A. M. D. (2008). Efeitos fisiológicos e evidências científicas da eficácia da fisioterapia aquática. Revista Movimenta, 1(1), 23-27. Recuperado em 15 fevereiro, 2017, de: http://www.nee.ueg.br/seer/index.php/movimenta/article/viewArticle/83.

Costa, D., Gonçalves, H. A., Lima, L. P., Ike, D., Cancelliero, K. M., \& Montebelo, M. I. L. (2010). Novos valores de referência para pressões respiratórias máximas na população brasileira. Jornal Brasileiro de Pneumologia, 36(3), 306-312. Recuperado em 05 junho, 2016, de: http://www.scielo.br/scielo.php?script=sci_arttext\&pid=S1806-37132010000300007.

Costa, D., Sampaio, L. M. M., Lorenzzo, V. A. P., Jamami, M., \& Damaso, A. R. (2003). Avaliação da força muscular respiratória e amplitudes torácicas e abdominais após a RFR em indivíduos obesos. Revista Latino-Americana de Enfermagem, 11(2), 156-160. Recuperado em 05 novembro, 2016, de: http://www.scielo.br/scielo.php?script=sci_arttext\&pid=S0104-11692003000200003. 
Costa, D., \& Jarnani, M. (2001). Bases fundamentais da espirometria. Revista Brasileira de Fisioterapia, 5(2), 95-102. Recuperado em 05 junho, 2016, de: http://www.rbfbjpt.org.br/article/51829c641ef1fa2b10000041.

Cruz, M. S. L., Fernandes, R. P., Reis, M. V. S., Policarpo, B. F., \& Filho, F. J. (2010). Efeitos de terapêuticas respiratórias e actividade física nas pressões respiratórias máximas de mulheres obesas. Motricidade, 6(2), 15-21. Recuperado em 18 junho, 2016, de: http://www.revistamotricidade.com/arquivo/2010_vol6_n2/v6n2a03.pdf.

De Almeida Fagundes, A., \& Da Silva, R. F. (2017). Efeitos da imersão em água aquecida sobre o sistema respiratório. Fisioterapia em Movimento, 19(4), 113-118. Recuperado em 18 dezembro, 2017, de: http://www2.pucpr.br/reol/public/7/archive/0007-00001823-ARTIGO_14.PDF.

Deturk, W. E., \& Cahalin, L. P. (2007). Fisioterapia cardiorrespiratória: Baseada em evidências. Porto Alegre, RS: Artmed.

Elias, R. G. M., Gonçalves, E. C. A., Moraes, A. C. F., Formaggio, C. M., \& Fernandes, C. A. M. (2012). Aptidão física funcional de idosos praticantes de hidroginástica. Revista Brasileira de Geriatria e Gerontologia, 15(1), 79-86. Recuperado em 11 junho, 2016, de: http://www.scielo.br/scielo.php?script=sci_arttext\&pid=S1809-98232012000100009.

Fechine, B. R. A., \& Trompieri, N. (2015). O processo de envelhecimento: as principais alterações que acontecem com o idoso com o passar dos anos. InterSciencePlace, 1(20), 106-194. Recuperado em 01 março, 2017, de: http://www.interscienceplace.org/isp/index.php/isp/article/view/196.

Freitas, E. R. F. S., Araújo, E. C. L. D., \& Alves, K. S. (2012). Influência do tabagismo na força muscular respiratória em idosos. Fisioterapia e Pesquisa, 19(4), 326-331. Recuperado em 26 junho, 2016, de: http://www.scielo.br/scielo.php?script=sci_arttext\&pid=S1809-29502012000400006.

Gonçalves, M. P., Tomaz, C. A. B., Cassiminho, A. L. F., \& Dutra, M. F. (2006). Avaliação da força muscular inspiratória e expiratória em idosas praticantes de atividade física e sedentárias. Revista Brasileira de Ciência e Movimento, 14(1), 37-44. Recuperado em 05 novembro, 2016, de: https://portalrevistas.ucb.br/index.php/RBCM/article/view/676.

Ide, M. R., Caromano, F. A., Dip, M. A. V. B., \& Guerino, M. R. (2007). Exercícios respiratórios na expansibilidade torácica de idosos: exercícios aquáticos e solo. Fisioterapia em Movimento, 20(2), 33-40. Recuperado em 03 junho, 2016, de: http://www2.pucpr.br/reol/pb/index.php/rfm?dd1=1557\&dd99=view\&dd98=pb.

Kruel, L. F. M. , Coertjens, M., Pinto, S. S., Alberton, C. L., Brentano, M. A. Efeito da Imersão sobre o Comportamento do Consumo de Oxigênio em Repouso. Revista Brasileira de Atividade Física e Saúde, 11, 25-31, 2006.

Nascimento, C., Trindade, S., Oliveira, M., Sousa, C., \& Abrahin, C. (2013). Efeitos dos exercícios resistidos nos indicadores de normalidade de força dos músculos respiratórios de idosos. FIEP BULLETIN, 83(1), 1-6. Recuperado em 17 março, 2018, de: http://www.fiepbulletin.net/index.php/fiepbulletin/article/view/2749/5353. 
Parreira, V. F., França, D. C., Zampa, C. C., Fonseca, M. M., Tomich, G. M. E., \& Britto, R. R. (2007). Pressões respiratórias máximas: Valores encontrados e preditos em indivíduos saudáveis. Revista Brasileira de Fisioterapia, 11(5), 361-368. Recuperado em 10 dezembro, 2016, de: http://www.scielo.br/scielo.php?script=sci_arttext\&pid=S1413-35552007000500006.

Pereira, C. A. C., Sato, T., \& Rodrigues, S. C. (2007). Novos valores de referência para espirometria forçada em brasileiros adultos de raça branca. Jornal Brasileiro de Pneumologia, 33(4), 397-406. Recuperado em 10 dezembro, 2016, de: http://www.scielo.br/scielo.php?script=sci_arttext\&pid=S1806-37132007000400008.

Pereira, F. D., Batista, W. O., Fuly, P. S. C., Junior, E. D. A., \& Silva, E. B. (2014). Atividade física e força muscular respiratória de idosos: uma revisão sistemática. Fisioterapia em Movimento, 27(1), 129-139. Recuperado em 03 junho, 2016, de: http://www.scielo.br/scielo.php?script=sci_arttext\&pid=S0103-51502014000100129.

Petroski, E. L. (2011). Antropometria: Técnicas e Padronizações. (5 $5^{\mathrm{a}}$ ed.). Várzea Paulista, SP: Fontoura.

Presto, B., \& Presto, L. D. N. (2009). Fisioterapia Respiratória. (4ª ed.). Rio de Janeiro, RJ: Editora Elsevier.

Ramos, R. A., \& Ferreira, A. S. (2014). Functional capacity in adults with hypertension as assessed by the six-minute walk distance test: systematic review. Fisioterapia e Pesquisa, 21(3), 257-263. Recuperado em 28 fevereiro, 2017, de: http://www.scielo.br/scielo.php?script=sci_arttext\&pid=S1809-29502014000300257.

Sarmento, G. J. V. (2010). Fisioterapia respiratória no paciente crítico: rotina clínicas. ( $3^{\mathrm{a}}$ ed.). São Paulo, SP: Manole.

Souza, H., Rocha, T., Pessoa, M., Rattes, C., Brandão, D., Fregonezi, G., Campos, S., Aliverti, A., \& Dornelas, A. (2014). Effects of inspiratory muscle training in elderly women on respiratory muscle strength, diaphragm thickness and mobility. Journals of Gerontology Series A: Biomedical Sciences and Medical Sciences,69(12), 1545-1553. Recuperado em 28 fevereiro, 2018, de: https://academic.oup.com/biomedgerontology/article/69/12/1545/593385.

Teixeira, C. S., Pereira, É. F., \& Rossi, A. G. (2007). A hidroginástica como meio para manutenção da qualidade de vida e saúde do idoso. Acta fisiátrica, 14(4), 226-232. Recuperado em 27 fevereiro, 2018, de: http://www.revistas.usp.br/actafisiatrica/article/view/102868/101159.

Tran, D., Rajwani, K., \& Berlin, D. A. (2018). Pulmonary effects of aging. Current Opinion in Anesthesiology, 31(1), 19-23. Recuperado em 27 fevereiro, 2018, de: https://journals.lww.com/coanesthesiology/Abstract/2018/02000/Pulmonary_effects_of_aging.5.aspx.

Recebido em 01/03/2017

Aceito em 30/06/2017 
Simone Mader Dall Agnol - Graduação em Fisioterapia. Especialista em Geriatria e Gerontologia. Mestranda do Programa de Pós-Graduação Stricto Sensu em Desenvolvimento Comunitário da Universidade Estadual do Centro-Oeste - Unicentro.

E-mail: monemader@hotmail.com

Thaisa Iohn da Silva - Graduação em Educação Física pela Faculdade Guairacá/SESG, Guarapuava-PR, Brasil.

E-mail: masca58@hotmail.com

Denise Barth Rebesco - Graduação em Educação Física. Especialista em Gestão em Saúde. Mestranda do Programa de Pós-Graduação Stricto Sensu em Desenvolvimento Comunitário da Universidade Estadual do Centro-Oeste - Unicentro.

E-mail: deniserebesco@gmail.com

André de Camargo Smolarek - Graduação em Educação Física. Especialista em Fisiologia do Exercício. Mestre em Educação Física. Professor Faculdade Guairacá/SESG, Guarapuava-PR, Brasil.

E-mail: andrecsk@gmail.com

William Cordeiro de Souza - Graduação em Educação Física. Especialista em Fisiologia do Exercício com Ênfase em Treinamento Desportivo.

E-mail: professor_williamsouza@yahoo.com.br

Meiriélly Furmann - Graduação em Fisioterapia. Mestranda do Programa de Pós-Graduação Stricto Sensu em Desenvolvimento Comunitário da Universidade Estadual do Centro-Oeste Unicentro.

E-mail: meiry.furmann2@gmail.com

Luis Paulo Gomes Mascarenhas - Graduação em Educação Física. Mestre em Atividade Física e Saúde. Doutor Saúde da Criança e do Adolescente. Professor do Programa de Pós-Graduação Stricto Sensu em Desenvolvimento Comunitário da Universidade Estadual do Centro-Oeste Unicentro.

E-mail: luismsk@gmail.com 